\title{
Analytic energy gradient for the projected Hartree-Fock method
}

\author{
Roman Schutski, ${ }^{1}$ Carlos A. Jiménez-Hoyos, ${ }^{1}$ and Gustavo E. Scuseria ${ }^{2}$ \\ ${ }^{1}$ Department of Chemistry, Rice University, Houston, Texas 77251-1892, USA \\ ${ }^{2}$ Department of Chemistry and Department of Physics and Astronomy, Rice University, Houston, \\ Texas 77251-1892, USA
}

(Received 13 February 2014; accepted 2 May 2014; published online 22 May 2014)

\begin{abstract}
We derive and implement the analytic energy gradient for the symmetry Projected Hartree-Fock (PHF) method avoiding the solution of coupled-perturbed HF-like equations, as in the regular unprojected method. Our formalism therefore has mean-field computational scaling and cost, despite the elaborate multi-reference character of the PHF wave function. As benchmark examples, we here apply our gradient implementation to the ortho-, meta-, and para-benzyne biradicals, and discuss their equilibrium geometries and vibrational frequencies. () 2014 AIP Publishing LLC. [http://dx.doi.org/10.1063/1.4876490]
\end{abstract}

\section{INTRODUCTION}

One of the most challenging problems for modern computational quantum chemistry is the accurate description of systems with strong or static correlation. These situations include, for example, the breaking of chemical bonds, nonequilibrium geometries, clusters with multiple metal centers, or states with ubiquitous multi-determinant character like biradicals. Standard methods based on a single reference determinant often fail to give even a qualitatively correct description of these cases marked by the presence of near degeneracies. Methods for improving the wave function in such situations have been developed over the years. A simple solution is to replace the single reference determinant by a multi-configuration expansion, which is the idea behind exact diagonalization over an active space. ${ }^{1}$ The selection of such an active space is often not unambiguous. When the strong correlation is associated with spin symmetry breaking, methods to automatically choose the corresponding active space have been proposed, ${ }^{2-4}$ but the problem cannot be considered fully solved.

In recent work, ${ }^{5}$ we have shown how a variationally optimized symmetry-projected state results in a multi-reference wave function that accurately describes static (or strong) correlations in finite systems. Traditional constructions of the Hilbert space are mostly based on particle-hole excitations out of a reference determinant, leading to a basis of orthonormal configurations. In PHF, on the other hand, a linear combination of non-orthogonal determinants generated by the action of rotation operators on the reference state is formed. Integration over these non-orthogonal states weighted by coefficients depending on the irreducible representation of interest is the key ingredient for symmetry restoration. ${ }^{6}$ In our work, we used the more powerful variation-after-projection (VAP) scheme, which implies that the broken-symmetry reference determinant is variationally optimized to minimize the projected energy. This is different from a simpler projectionafter-variation (PAV) scheme ${ }^{7}$ traditionally associated with projection based methods in quantum chemistry. Symmetry implies degeneracy and degeneracy is at the root of the static correlation problem. In this sense, the PHF methodology is able to account for the static correlations due to symmetries present in finite systems.

It is well known that allowing the HF determinant to break physical symmetries leads to a better estimate of the energy in situations where near-degeneracies are present. ${ }^{8}$ Near-degeneracies can be easily diagnosed in a symmetry restricted HF (RHF) treatment by closure of the HOMO-LUMO energy gap, which in turn leads to spontaneous symmetry breaking. ${ }^{9}$ For instance, Unrestricted HF (UHF) predicts qualitatively correct single bond dissociation curves in contrast to RHF. However, at dissociation the UHF wave function is not an eigenfunction of the $S^{2}$ operator. Spin symmetry breaking occurs spontaneously in bond breaking processes (leading to open-shell fragments) and even though it is an artifact resulting from the approximate nature of the wave function, it definitely accounts for important static correlation, a fact termed "symmetry dilemma" 10 many years ago. A Generalized HF (GHF) broken symmetry solution where space, spin, and complex conjugation symmetries are all broken, is the most flexible wave function obtainable using a single determinant. ${ }^{8}$ Despite being more flexible, GHF solutions are not necessarily better or ideal, as they may display kinks in the potential energy surface. ${ }^{11}$ The machinery of selfconsistent symmetry breaking and restoration remedies these maladies.

PHF theory deliberately breaks the symmetries of the reference determinant $|\Phi\rangle$ whose energy is optimized in the presence of symmetry projection operators $\hat{P}$,

$$
E=\frac{\left\langle\Phi\left|\hat{P}^{\dagger} H \hat{P}\right| \Phi\right\rangle}{\left\langle\Phi\left|\hat{P}^{\dagger} \hat{P}\right| \Phi\right\rangle}=\frac{\langle\Phi|H \hat{P}| \Phi\rangle}{\langle\Phi|\hat{P}| \Phi\rangle}
$$

Here, we have used the fact that the symmetry projection operator $\hat{P}$ commutes with the Hamiltonian and is idempotent. The resulting wave function $P|\Phi\rangle$ has an elaborate multi-determinant structure that recovers a significant portion of static correlation. The PHF wave function yields 
correct quantum numbers and preserves the physical symmetries of the system, while still being fully determined by a single set of occupied molecular orbitals. The specific nature of the PHF wave function depends on the particular symmetries of the underlying HF reference determinant that are broken and restored in each case. More than one symmetry can be deliberately broken and restored using PHF. The group-theoretical classification of all possible classes of Slater determinants (solutions to the HF equations) was first given by Fukutome ${ }^{12}$ and later by Stuber and Paldus. ${ }^{13}$ The molecular Hamiltonian is number conserving and preserves total spin symmetry (broken in UHF), $S_{z}$ symmetry (broken in non-collinear GHF), complex conjugation symmetry (broken by allowing molecular orbitals (MOs) to become complex) and point group symmetry (often broken in UHF solutions). Additionally, number is a symmetry of the Hamiltonian that can be broken if working with determinants built from quasiparticles albeit never spontaneously for Coulombic repulsive systems. In previous work, we have dealt with symmetry breaking and restoration of number, spin (both collinear and non-collinear), molecular point group, complex conjugation, and full space group in lattice models with periodic boundary conditions. ${ }^{14-16} \mathrm{We}$ here focus on molecules where translation is not a symmetry and limit ourselves to spin symmetry breaking, although the PHF formalism and its analytic energy gradient are effectively the same in the case of point group and other symmetries.

The PHF wave function inherits several advantages from the HF approach. The method is fully variational, which facilitates the evaluation of properties such as energy derivatives. Another advantage is its computational scaling: the PHF procedure scales with the number of basis functions in the same way the regular HF method does. ${ }^{17}$ However, some drawbacks are present. The need of an integration grid makes PHF more expensive than HF. As has been thoroughly discussed, ${ }^{5,18}$ PHF is neither size consistent nor size extensive. This means that in the thermodynamic limit, PHF reverts to HF. Despite its accuracy for small systems, there are types of correlations (unrelated to symmetries) that PHF does not recover efficiently. These residual correlations are mostly "dynamical" in nature and can be accounted for using a multi-component PHF approach. ${ }^{19}$ Nevertheless, because of its black-box description of static correlation, PHF is an excellent starting point for subsequent treatment of residual correlation via, for example, Quantum Monte Carlo. ${ }^{20}$

Availability of analytic energy derivatives is of outmost importance for a method to become of practical use. In this paper, we present the derivation and implementation of the PHF analytical energy gradient. A previous attempt by Handy and Rice ${ }^{21}$ was limited to the spin-projected HF method (or Extended HF (EHF)), ${ }^{10,22}$ which may be viewed as a special case of the more general PHF formalism, and was based on Löwdin's many-body spin projection operator (as opposed to the one-body symmetry rotation operators we use). The resulting mathematical formulation of EHF gradients ${ }^{21}$ is quite different from ours, and the results of Handy and Rice are not directly applicable to the more general PHF methodology discussed here.

\section{THEORY}

Before proceeding with the analytic energy derivatives we describe the formalism of the PHF method. In contrast to our previous work ${ }^{5}$ where PHF was formulated in terms of the density matrix of the broken-symmetry determinant, we here choose to formulate it in terms of molecular orbital coefficients, as first done by Schmid. ${ }^{23}$ This section is organized as follows. First, we introduce the notation used for the projectors and review their basic properties (Sec. II A). We formulate the PHF equations in a non-orthogonal basis using molecular orbitals in Sec. II B. Finally, we present the expressions for the analytic energy gradients and comment on our implementation (Secs. II C and II D).

\section{A. Projectors and rotation operators}

Let us introduce an ansatz for the wave function that satisfies specific chosen symmetries (labelled by the quantum numbers $j$ and $m$ ):

$$
\left|\Psi_{m}^{j}\right\rangle=\sum_{k} f_{k} \hat{P}_{m k}^{j}|\Phi\rangle
$$

The function $|\Phi\rangle$ is a broken-symmetry Slater determinant $|\Phi\rangle$ and $\hat{P}_{m k}^{j}$ is a "projection" operator for arbitrary groups. Here, $j$ labels the irreducible representations (irreps), while $m(k)$ denote the rows (columns) of the irrep. The linear variational coefficients $f_{k}$ are introduced in order to remove unphysical dependencies of $\left|\Psi_{m}^{j}\right\rangle$ on the orientation of $|\Phi\rangle .{ }^{24}$ Note that for Abelian groups the linear combination above reduces to the standard PHF expression introduced by Löwdin. ${ }^{10}$ For continuous groups the projection operators can be written in the standard form: ${ }^{25}$

$$
\hat{P}_{m k}^{j}=\frac{1}{V} \int_{V} d \theta \omega_{m k}^{j}(\theta) \hat{R}(\theta),
$$

where $\theta$ denotes the element of the group. Additionally, $V$ is the volume of integration, $\omega_{m k}^{j}(\theta)$ are integration weights (characters) of the irrep and $\hat{R}(\theta)$ is a rotation operator. The integral above should be understood as a summation for discrete groups. More details on the projection operators can be found in Ref. 26. The operators $\hat{P}_{m k}^{j}$ satisfy the properties

$$
\begin{gathered}
\left(\hat{P}_{m k}^{j}\right)^{\dagger}=\hat{P}_{k m}^{j}, \\
\hat{P}_{m k}^{j} \hat{P}_{k^{\prime} m^{\prime}}^{j^{\prime}}=\hat{P}_{m m^{\prime}}^{j} \delta_{j j^{\prime}} \delta_{k k^{\prime}} .
\end{gathered}
$$

The molecular orbitals $|i\rangle$ determining the wave function $|\Phi\rangle$ are represented by a linear combinations of basis functions in the form

$$
|i\rangle=\sum_{\mu} C_{\mu i}|\mu\rangle .
$$

They are assumed to be orthonormal,

$$
\langle i \mid j\rangle=\delta_{i j}=\left(C^{\dagger} S C\right)_{i j},
$$

where $S$ is the overlap matrix between basis functions. Let us now consider the action of the (single-particle) rotation 
operator $\hat{R}(\theta)$ on the molecular orbital $|i\rangle$,

$$
|i(\theta)\rangle \equiv \hat{R}(\theta)|i\rangle=\sum_{\mu \nu \lambda}|\mu\rangle S_{\mu \nu}^{-1} R_{\nu \lambda}(\theta) C_{\lambda i},
$$

where $R_{\mu \nu}(\theta) \equiv\langle\mu|\hat{R}(\theta)| v\rangle$ is an element of the rotation matrix in the atomic basis set. We should note, however, that $S^{-1} R(\theta)$ is independent of the overlap between basis functions, a fact that we use later on in our derivation.

\section{B. Variational conditions and PHF equations}

Using the ansatz of Eq. (2), the energy corresponding to a state with quantum number $j$ can be written as

$$
E^{j}=\sum_{k k^{\prime}} f_{k}^{*} f_{k^{\prime}} H_{k k^{\prime}},
$$

where we have introduced the Hamiltonian matrix elements

$$
H_{k k^{\prime}}=\left\langle\Phi\left|H \hat{P}_{k k^{\prime}}^{j}\right| \Phi\right\rangle \text {. }
$$

In the same way, we also define norm matrix elements associated with the projected state as

$$
N_{k k^{\prime}}=\left\langle\Phi\left|\hat{P}_{k k^{\prime}}^{j}\right| \Phi\right\rangle .
$$

The Hamiltonian and the norm matrices can be proved to be Hermitian using the properties of the projectors. We impose two variational conditions on the wave function,

$$
\begin{gathered}
f^{\dagger} N f=1, \\
C^{\dagger} S C=1_{N \times N},
\end{gathered}
$$

where the former equation constitutes a normalization of the full PHF wave function, while the latter equation characterizes the orthonormality of the occupied molecular orbitals.

In order to evaluate the matrix elements in Eqs. (8) and (10), we write them in integral form as

$$
\begin{gathered}
H_{k k^{\prime}}=\int d \theta \omega_{k k^{\prime}}^{j}(\theta) n(\theta) h(\theta), \\
N_{k k^{\prime}}=\int d \theta \omega_{k k^{\prime}}^{j}(\theta) n(\theta), \\
h(\theta)=\frac{\langle\Psi|H \hat{R}(\theta)| \Psi\rangle}{\langle\Psi|\hat{R}| \Psi\rangle}, \\
n(\theta)=\langle\Psi|\hat{R}(\theta)| \Psi\rangle .
\end{gathered}
$$

The overlap kernel can be evaluated using Wick's theorem as

$$
n(\theta)=\operatorname{det}_{N}\left(C^{\dagger} R(\theta) C\right)=\operatorname{det}_{N}(M(\theta)),
$$

where the notation $\operatorname{det}_{N}$ emphasizes that the determinant is taken only over the block of occupied orbitals. That is, $C$ is the rectangular matrix of occupied orbitals, rendering $M(\theta)$ as an $N \times N$ matrix, with $N$ being the number of occupied orbitals.

The Hamiltonian kernel can be evaluated using a generalized Wick's theorem (see, e.g., Ref. 27). The final expression is given by

$$
\begin{gathered}
h(\theta)=\sum_{\mu \nu}\left(\langle\mu|h| v\rangle+\frac{1}{2} G_{\mu \nu}(\theta)\right) \rho_{\nu \mu}(\theta), \\
G_{\mu \nu}(\theta)=\sum_{\lambda \sigma}\langle\mu \lambda|\hat{v}| \nu \sigma\rangle \rho_{\sigma \lambda}(\theta),
\end{gathered}
$$

where $\langle\mu|h| v\rangle$ are core Hamiltonian (one-electron) integrals and $\langle\mu \lambda|\hat{v}| \nu \sigma\rangle$ are (antisymmetrized) two-electron integrals in Dirac notation. Here, the transition density matrix $\rho(\theta)$, is given by

$$
\rho(\theta)=S^{-1} R(\theta) C M^{-1}(\theta) C^{\dagger} .
$$

Only the occupied orbitals are used in the expression above ( $C$ is thus a rectangular matrix).

We turn to presenting the PHF equations using the notation given above. Following the variational conditions (11a) and (11b), we introduce the Lagrangian

$$
\begin{aligned}
L^{j}[f, C]= & \sum_{k k^{\prime}} f_{k}^{*} f_{k^{\prime}} H_{k k^{\prime}}-\sum_{i j} \epsilon_{i j}\left[C^{\dagger} S C-1_{N \times N}\right]_{j i} \\
& -E^{j}\left(f^{\dagger} N f-1\right) .
\end{aligned}
$$

Setting $\frac{\partial L^{j}}{\partial f_{k}}=0$ leads to a generalized eigenvalue problem for the linear coefficients $\{f\}$,

$$
H_{k k^{\prime}} f_{k^{\prime}}=E^{j} N_{k k^{\prime}} f_{k^{\prime}} .
$$

In the same way, we handle the expressions for $\frac{\partial L^{j}}{\partial C_{\alpha i}}$ and $\frac{\partial L^{j}}{\partial C_{\alpha i}^{*}}$ to arrive at the following PHF equations:

$$
\begin{aligned}
& \sum_{k k^{\prime}} f_{k}^{*} f_{k^{\prime}} \int d \theta \omega_{k k^{\prime}}^{j}(\theta) n(\theta) \sum_{\mu \nu} W_{i \mu}(\theta)\left\{\left(h(\theta)-E^{j}\right) \delta_{\mu \nu}\right. \\
& \left.+X_{\mu \nu}(\theta)\right\} R_{\nu \alpha}(\theta)=\left[\epsilon C^{\dagger} S\right]_{i \alpha}, \\
& \sum_{k k^{\prime}} f_{k}^{*} f_{k^{\prime}} \int d \theta \omega_{k k^{\prime}}^{j}(\theta) n(\theta) \sum_{\mu}\left\{\left(h(\theta)-E^{j}\right) \delta_{\alpha \mu}\right. \\
& \left.\quad+Y_{\alpha \mu}(\theta)\right\} Z_{\mu i}(\theta)=[S C \epsilon]_{\alpha i},
\end{aligned}
$$

where we have defined

$$
\begin{gathered}
W_{i \mu}=\left[M^{-1}(\theta) C^{\dagger}\right]_{i \mu} \\
X_{\mu \nu}(\theta)=\sum_{\lambda \sigma}\left(\langle\mu|h| \lambda\rangle+G_{\mu \lambda}(\theta)\right) S_{\lambda \sigma}^{-1}\left(\delta_{\sigma \nu}-[S \rho(\theta)]_{\sigma \nu}\right), \\
Y_{\alpha \mu}(\theta)=\sum_{\lambda \sigma}\left(\delta_{\alpha \lambda}-[S \rho(\theta)]_{\alpha \lambda}\right)\left(\langle\lambda|h| \sigma\rangle+G_{\lambda \sigma}(\theta)\right) S_{\sigma \mu}^{-1}, \\
Z_{\mu i}=\left[R(\theta) C M^{-1}(\theta)\right]_{\mu i} .
\end{gathered}
$$

As one may expect, these equations are adjoints of each other. However, in contrast to the conventional Hartree-Fock scheme, the matrix of Lagrange multipliers $\epsilon$ cannot be associated with orbital energies. ${ }^{23}$ Moreover, it completely vanishes at convergence (to prove it one has to multiply the first of Eqs. (18) by $C$ from the left (or the second of Eqs. (18) by $\mathrm{C}^{\dagger}$ from the right) and work out the definitions of the appropriate quantities)

$$
\epsilon_{i j}=0 \quad \forall i, j=1, \ldots, N .
$$


We also note that the PHF equations (Eqs. (18)) are not eigenvalue equations. This formulation is thus somehow inconvenient in a practical optimization of the PHF wave function.

\section{First energy derivatives}

Having expressed the PHF equations in terms of atomic integrals and molecular orbital coefficients (of the brokensymmetry determinant), we can derive an expression for the first derivative of the energy. Our procedure closely follows the construction of energy derivatives of the standard HF method, which stemmed from works of Bratoz, ${ }^{28}$ and later by Pulay ${ }^{29}$ and Gerrat and Mills. ${ }^{30}$ For a review of the history of analytic gradients, see Ref. 31 .

In order to obtain the analytic derivative one differentiates the energy expression (Eq. (8)) with respect to the parameter $x$. To eliminate the derivatives of various parameters of the wave function from the final expression we consequently use the variational conditions.

Differentiation of Eq. (11a) leads to

$$
\begin{gathered}
\sum_{k k^{\prime}}\left[\frac{\partial f_{k}^{*}}{\partial x} f_{k^{\prime}} N_{k k^{\prime}}+\frac{\partial f_{k^{\prime}}}{\partial x} f_{k}^{*} N_{k k^{\prime}}+f_{k}^{*} f_{k^{\prime}} \frac{\partial N_{k k^{\prime}}}{\partial x}\right]=0 \\
\Rightarrow \sum_{k k^{\prime}}\left[\frac{\partial f_{k}^{*}}{\partial x} f_{k^{\prime}}+\frac{\partial f_{k^{\prime}}}{\partial x} f_{k}^{*}\right] N_{k k^{\prime}}=-\sum_{k k^{\prime}} f_{k}^{*} f_{k^{\prime}} \frac{\partial N_{k k^{\prime}}}{\partial x} .
\end{gathered}
$$

Differentiation of the orthonormality condition of the occupied molecular orbitals (see Eq. (11b)) leads to

$$
\begin{aligned}
& \frac{\partial C_{\mu i}^{*}}{\partial x} S_{\mu \nu} C_{\nu j}+C_{\mu i}^{*} S_{\mu \nu} \frac{\partial C_{\nu j}}{\partial x}+C_{\mu i}^{*} \frac{\partial S_{\mu v}}{\partial x} C_{\nu j}=0 \\
& \Rightarrow \frac{\partial C_{\mu i}^{*}}{\partial x} S_{\mu \nu} C_{\nu j}+C_{\mu i}^{*} S_{\mu \nu} \frac{\partial C_{\nu j}}{\partial x}=-C_{\mu i}^{*} \frac{\partial S_{\mu v}}{\partial x} C_{\nu j},
\end{aligned}
$$

which relates the derivatives of the molecular orbital coefficients to derivatives of the overlap matrix.

We are now in a position to take the derivative of the full energy expression. Using Eq. (8), we arrive at

$$
\frac{\partial E^{j}}{\partial x}=\sum_{k k^{\prime}}\left[\frac{\partial f_{k}^{*}}{\partial x} f_{k^{\prime}}+f_{k}^{*} \frac{\partial f_{k^{\prime}}}{\partial x}\right] H_{k k^{\prime}}+f_{k}^{*} f_{k^{\prime}} \frac{\partial H_{k k^{\prime}}}{\partial x} .
$$

By using Eqs. (21) and (17), we eliminate $\frac{\partial f}{\partial x}$ from the expression above (Eq. (23)):

$$
\frac{\partial E^{j}}{\partial x}=\sum_{k k^{\prime}} f_{k}^{*} f_{k^{\prime}}\left[\frac{\partial H_{k k^{\prime}}}{\partial x}-E^{j} \frac{\partial N_{k k^{\prime}}}{\partial x}\right] .
$$

Standard differentiation and some algebraic manipulations can be carried out to arrive at

$$
\begin{aligned}
& \left(\frac{\partial H_{k k^{\prime}}}{\partial x}-E^{j} \frac{\partial N_{k k^{\prime}}}{\partial x}\right)=\int d \theta \omega_{k k^{\prime}}^{j}(\theta) n(\theta) \\
& \quad \times\left\{\sum_{\mu \nu}\left[\langle\mu|h| v\rangle^{x} \rho_{\nu \mu}(\theta)+G_{\mu \nu}^{x}(\theta) \rho_{\nu \mu}(\theta)\right]\right. \\
& \quad+\left(h(\theta)-E^{j}\right) \sum_{\mu \nu} S_{\mu \nu}^{x} \rho_{\nu \mu}(\theta)
\end{aligned}
$$

$$
\begin{aligned}
& -\sum_{\mu \nu \lambda \sigma} S_{\mu \nu}^{x} \rho_{\nu \lambda}(\theta)\left[\langle\lambda|h| \sigma\rangle+G_{\lambda \sigma}(\theta)\right] \rho_{\sigma \mu}(\theta) \\
& +\sum_{\mu \nu \alpha i}\left[W_{i \mu}(\theta)\left(\left(h(\theta)-E^{j}\right) \delta_{\mu \nu}+X_{\mu \nu}(\theta)\right) R_{\nu \alpha}(\theta)\right] \frac{\partial C_{\alpha i}^{*}}{\partial x} \\
& \left.+\sum_{\mu \alpha i}\left[\left(h(\theta)-E^{j}\right) \delta_{\alpha \mu}+Y_{\alpha \mu}(\theta)\right] Z_{\mu i}(\theta) \frac{\partial C_{\alpha i}}{\partial x}\right\}
\end{aligned}
$$

where we defined

$$
\begin{gathered}
S_{\mu \nu}^{x}=\frac{\partial\langle\mu \mid v\rangle}{\partial x}, \\
h_{\mu \nu}^{x}=\frac{\partial\langle\mu|h| v\rangle}{\partial x}, \\
G_{\mu \nu}^{x}(\theta)=\sum_{\lambda \sigma} \frac{\partial\langle\mu \lambda|\hat{v}| \nu \sigma\rangle}{\partial x} \rho_{\sigma \lambda}(\theta) .
\end{gathered}
$$

We then use the PHF equations (18) and equation (22) to eliminate $\frac{\partial C_{\alpha i}}{\partial x}$ and $\frac{\partial C_{\alpha i}^{*}}{\partial x}$ from the expression above:

$$
\begin{aligned}
& \left(\frac{\partial H_{k k^{\prime}}}{\partial x}-E^{j} \frac{\partial N_{k k^{\prime}}}{\partial x}\right)=\int d \theta \omega_{k k^{\prime}}^{j}(\theta) n(\theta) \\
& \quad \times\left\{\sum_{\mu \nu}\left[\langle\mu|h| \nu\rangle^{x} \rho_{\nu \mu}(\theta)+G_{\mu \nu}^{x}(\theta) \rho_{\nu \mu}(\theta)\right]\right. \\
& \quad+\left(h(\theta)-E^{j}\right) \sum_{\mu \nu} S_{\mu \nu}^{x} \rho_{\nu \mu}(\theta) \\
& \quad-\sum_{\mu \nu \lambda \sigma} S_{\mu \nu}^{x} \rho_{\nu \lambda}(\theta)\left[\langle\lambda|h| \sigma\rangle+G_{\lambda \sigma}(\theta)\right] \rho_{\sigma \mu}(\theta) \\
& \left.\quad+\sum_{i j}\left[C^{\dagger} S^{x} C\right]_{i j} \epsilon_{j i}\right\} .
\end{aligned}
$$

We note that at convergence the matrix of Lagrange multipliers vanishes (Eq. (20)), and this gives the final expression for the analytic derivative

$$
\begin{aligned}
& \left(\frac{\partial H_{k k^{\prime}}}{\partial x}-E^{j} \frac{\partial N_{k k^{\prime}}}{\partial x}\right)=\int d \theta \omega_{k k^{\prime}}^{j}(\theta) n(\theta) \\
& \quad \times\left\{\sum_{\mu \nu}\left[\langle\mu|h| \nu\rangle^{x} \rho_{\nu \mu}(\theta)+G_{\mu \nu}^{x}(\theta) \rho_{\nu \mu}(\theta)\right]\right. \\
& +\left(h(\theta)-E^{j}\right) \sum_{\mu \nu} S_{\mu \nu}^{x} \rho_{\nu \mu}(\theta) \\
& \left.\quad-\sum_{\mu \nu \lambda \sigma} S_{\mu \nu}^{x} \rho_{\nu \lambda}(\theta)\left[\langle\lambda|h| \sigma\rangle+G_{\lambda \sigma}(\theta)\right] \rho_{\sigma \mu}(\theta)\right\} .
\end{aligned}
$$

Equation (28) does not contain the derivatives of the wave function, and there is no need to solve coupled-perturbed-like equations. This fact, which is a consequence of the fully variational nature of the PHF wave function, was emphasized already in the very first works on HF gradients. ${ }^{29,30}$ We note that our expression, indeed, reduces to the $\mathrm{HF}$ derivative in the case of a trivial projector (see Appendix A).

\section{Remarks on implementation}

The method described in this paper has been implemented in a development version of the Gaussian 
software package. ${ }^{32}$ Our implementation was extensively verified against numerical differentiation results. Because of the relatively involved final expression, it may be valuable to describe how we have organized our code. One may proceed as follows (refer to Eq. (28)):

1. The wave function parameters $(C, f)$ and the total energy $E^{j}$ are obtained from the PHF optimization. The space of integration parameters $\theta$ (Eq. (3)) is discretized.

2. Atomic integrals and their derivatives are calculated: $S$, $S^{x},\langle\mu|h| v\rangle,\langle\mu|h| \nu\rangle^{x},\langle\mu \nu|\hat{v}| \lambda \sigma\rangle,\langle\mu \nu|\hat{v}| \lambda \sigma\rangle^{x}$.

3. For each value of $\theta$ the transition density matrix $\rho(\theta)$ is built.

4. The overlap terms $n(\theta)$ are calculated.

5. The transition density matrices are contracted with atomic integrals to give two-body terms $G(\theta), G(\theta)^{x}$.

6. The transition density matrices are contracted with one body atomic integrals and two-body terms to form $h(\theta)$ and $\rho(\theta)(h+G(\theta)) \rho(\theta)$ and complete the expressions under the integral in Eq. (28).

7. Finally, one performs a contraction of the integration weights $\omega_{k k^{\prime}}(\theta)$ with $\{f\}$ and a numerical integration over $\theta$.

The most expensive part of the procedure is the calculation of the two-electron integrals and, especially, their derivatives, which scales as $M^{4}$ in small systems, where $M$ is the number of basis functions, and as $M^{2}$ asymptotically. ${ }^{17}$ In the algorithm described above, the operations on each grid point are independent, and it is trivial to run them in parallel. The evaluation of the energy (or energy gradient) of the Projected HF wavefunction is roughly $N_{\text {grid }}$ times more expensive than a corresponding HF calculation. For SUHF, it is $2 \times N_{\text {grid }}$; the factor of 2 is due to the fact that the transition density matrices $\rho(\theta)$ have non-vanishing mixed-spin components. The number of grid points required depends weakly (sub-linearly) on the size of the subspace where spin symmetry-breaking occurs.

\section{RESULTS}

Earlier applications of PHF to molecular systems before our recent work are very limited. Rosenberg and Martino ${ }^{33}$ and Klimo and Tin̆o ${ }^{34}$ used spin-projected HF to study small molecules and radicals. Karadakov and Cooper ${ }^{35}$ performed self-consistent spin-projected UHF calculations on polyenes using an implementation based on spin-coupled valence bond theory. Recently, PHF was used by Samanta et $a l .{ }^{36}$ to study the bare copper oxide cores and by Rivero et $a l .{ }^{37,38}$ to study singlet-triplet (ST) splittings in various molecules and the polyradical character in polyacene systems.

We have chosen the $o^{-}, m^{-}$, and $p$-benzyne biradicals as benchmark examples to test our PHF gradient implementation. The proper description of these molecules is quite challenging because strong correlation effects are usually present in biradicals. For example, to accurately describe the lowest singlet and the lowest triplet states with $m=0$, where $m$ is the eigenvalue of $S_{z}$, one needs at least a two-configuration wave function. In contrast, a single-configuration wave func- tion is a good approximation for the lowest triplet states with $m= \pm 1.39$

The benzyne isomers have been well studied, both experimentally and theoretically. The interest on benzynes is motivated by the possible role of $p$-benzyne in the effect of antitumor drugs and by the identification of $o$-benzyne as an intermediate in elimination reactions. We point the reader to Ref. 40 for a detailed discussion of the biradical character in benzyne molecules. Biradical systems in general have become popular targets for testing novel electronic structure methods. ${ }^{41,42}$ Recent theoretical studies on benzynes have primarily concentrated on the geometry and the biradical character of the ground state, ${ }^{40,43}$ the ST separations, ${ }^{40,44,45}$ and the vibrational spectra. ${ }^{43,46,47}$

In this work, we study the optimized geometries and vibrational frequencies of the benzyne isomers with projected HF methods and compare them with the Complete Active Space Self-Consistent Field (CASSCF) method and experimental data when available.

\section{A. Computational details}

Collinear (UHF-type) and non-collinear (GHF-type) reference functions were used in our calculations. We follow the notation introduced in Ref. 5. If the HF wave function is collinear and breaks $S^{2}$ symmetry we denote the method as SUHF. If complex conjugation symmetry is also broken and restored, we refer to this case as KSUHF. The same notation is used for GHF-based methods by replacing U with G. All computations were carried out using an augmented split-valence 6-311G(p,d) basis set with six Cartesian components for $d$ functions. This particular choice was made to better match the results of previous studies on benzynes. The quality of PHF results has a weak ${ }^{5}$ dependence on the basis set size (similar to CASSCF), which is consistent with the method's ability to describe static, as opposed to dynamic, correlation. The integration grid for the gauge angle $\theta$ (see Eq. (3)) of the projection operator is chosen to provide the correct expectation value of $S^{2}$ to a precision of $10^{-7}$ or better (12 points were used for $S^{2}$ projection on an UHF reference and $7 \times 12 \times 7$ for full triaxial spin projection on a GHF reference). The harmonic frequencies from PHF were obtained using a four point numerical differentiation of first order analytic derivatives. For other methods, analytic second derivatives were used when available.

We have considered the possibility that there are multiple solutions to the HF and PHF equations. To this end, we have ensured that our HF solutions (i.e., our UHF calculations) are stable. Although this cannot, in principle, guarantee that the global minimum energy wave function is obtained, our HF energies and optimized geometries agree with the results of other authors. ${ }^{46,48}$ The lowest energy stable HF determinant has been used as an initial guess for the PHF procedure. Although this strategy does not necessarily lead to the lowestenergy PHF state (or even a local minimum), we have found that it often does. A stability analysis of the PHF state can be performed, but this is out of the scope of the present study.

The active space of the CASSCF calculations consisted of 8 electrons in 8 orbitals, as previously proposed by various 
authors. ${ }^{49,50}$ The choice of this active space is also supported by our UHF calculations (see Appendix B). We chose the active space based on the natural orbital occupancy. ${ }^{3}$ The $(8,8)$ active space corresponds to the inclusion of all orbitals with occupation numbers roughly differing by 0.02 from double or zero occupation.

\section{B. Adiabatic singlet-triplet separations}

The different benzyne isomers are known to display varying degrees of biradical character. ${ }^{42}$ All isomers have singlet ground states. Experimental ${ }^{51,52}$ ST separations change in the order

$$
\text { ortho }\left(37.5 \mathrm{kcal} \mathrm{mol}^{-1}\right)<\text { meta }\left(21.0 \mathrm{kcal} \mathrm{mol}^{-1}\right)<\text { para }(3.8
$$$$
\text { (2.1) } \mathrm{kcal} \mathrm{mol}^{-1} \text { ). }
$$

As it has been extensively discussed, ${ }^{40,44}$ static correlation is pervasive in benzynes. The smaller the ST gap, the larger the static correlation present in the singlet ground state. Table I lists the adiabatic ST splittings in $o-, m$-, and $p$ benzyne biradicals predicted by several methods.

Several conclusions can be drawn from the calculated ST splittings. First, we note that UHF predicts the correct ground state for $o$ - and $p$-benzyne, but not for $m$-benzyne. Also, as one may expect, UHF produces highly spin contaminated solutions for singlet states. For triplet states the spin contamination is smaller but still significant.

SUHF recovers a significant correlation energy (over $\mathrm{UHF}$ ) for the singlet states of all isomers. In contrast, triplet states are improved to a lesser degree, which leads to an overestimation of the ST splittings. Our results thus make evident the fact that the improvement that PHF provides over UHF is not the same for states of different symmetries, as the character of the correlations present (static vs. dynamic) need not be the same. We note that by breaking and restoring further symmetries of the wave function, for example $S_{z}$ symmetry with SGHF, one can systematically improve the quality of PHF wave functions. We point the reader to Ref. 37 for more examples of ST splittings calculated using PHF methods. For states with a significant fraction of static correlation, such as the ground state of the benzyne isomers, PHF yields a highquality wave function considering its fully variational nature and mean-field scaling. This conclusion is consistent with our recent findings regarding the quality of PHF wave functions in post-PHF quantum Monte Carlo calculations. ${ }^{20}$

TABLE I. Adiabatic ST-splittings $E(T)-E(S)\left(\mathrm{kcal} \mathrm{mol}^{-1}\right)$ in $o-, m$-, and $p$-benzyne as predicted by different methods.

\begin{tabular}{lccc}
\hline \hline Method & $p$-benzyne & $m$-benzyne & $o$-benzyne \\
\hline UHF & 11.1 & -6.0 & 20.4 \\
SUHF & 29.3 & 4.4 & 67.0 \\
KUHF & 23.4 & 6.9 & 50.6 \\
CASSCF(8,8) & 2.7 & 15.2 & 35.1 \\
Expt. $^{a}$ & 3.8 & 21.0 & 37.5 \\
$\left\langle S^{2}\right\rangle_{U H F}$ (singlet) & 1.80 & 1.31 & 1.59 \\
$\left\langle S^{2}\right\rangle_{U H F}$ (triplet) & 2.40 & 2.80 & 2.41 \\
\hline \hline
\end{tabular}

${ }^{\mathrm{a}}$ From photoelectronic experiments (Ref. 52).

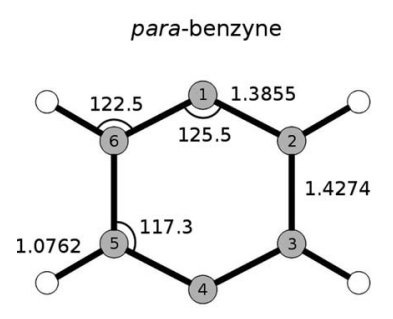

$E=-229.529159$
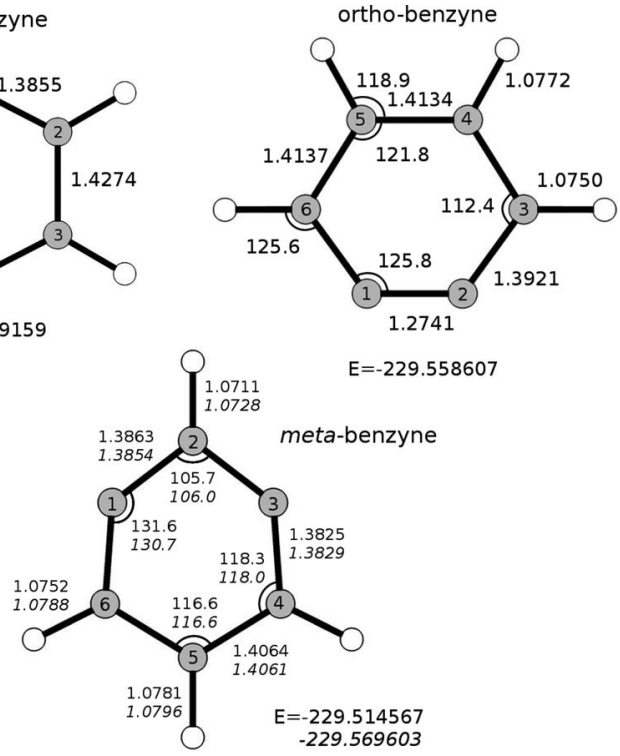

FIG. 1. Geometries of benzyne isomers as obtained with SUHF. For metabenzyne, the SGHF geometry is also given in italics. Absolute energies are in a.u.

\section{Optimized geometries}

We have used spin-projected HF to optimize singlet geometries of three benzyne isomers. The SUHF equilibrium geometries are shown in Fig. 1.

For all three isomers, the SUHF and CASSCF predicted equilibrium geometries are close. In almost every case SUHF predicts shorter bonds than CASSCF, rendering the optimized geometries closer to recent multi-reference CCSD(T) calculations. ${ }^{47}$ We emphasize that the difference in the bond lengths $\mathrm{C}_{1}-\mathrm{C}_{2}$ and $\mathrm{C}_{2}-\mathrm{C}_{3}$, which has been used by Crawford et al. $^{43}$ as a measure of diradical character, is $0.042 \AA$. This agrees with the results of Li and Paldus $(0.060 \AA)$ and our own CASSCF calculations $(0.035 \AA)$, indicating that the strong correlation effects are successfully captured by PHF.

In the case of $m$-benzyne, a SUHF stationary point with $C_{2 v}$ symmetry corresponds to a first order saddle point. The actual SUHF minimum has only $C_{s}$ symmetry and is $18.8 \mathrm{kcal} \mathrm{mol}^{-1}$ lower in energy. We note that the lowest energy stable UHF solution also has a $C_{s}$ minimum. SUHF is thus unable in this case to restore the broken spatial symmetry in the UHF wave function. Using a non-collinear GHFtype determinant, $\mathrm{HF}$ yields a minimum structure with $C_{2 v}$ symmetry. The SGHF equilibrium geometry compares nicely with CASSCF and also possesses $C_{2 v}$ symmetry. Indeed, the problem displayed by UHF-type determinants has been previously observed in density functional theory ${ }^{53}$ (DFT) calculations on small symmetric systems, ${ }^{54}$ and can be partially explained by spin frustration effects. Finally, we emphasize that all our PHF calculations correctly predict a monocyclic equilibrium structure (see Refs. 46 and 55 for a discussion on this issue) for $m$-benzyne. For example, the angle $\mathrm{C}_{1}-\mathrm{C}_{2}-\mathrm{C}_{3}$ in the SUHF and SGHF optimized structures is $105.7^{\circ}$ and $106.0^{\circ}$, respectively.

The SUHF equilibrium structure of $o$-benzyne is sufficiently close to the experimental results of Groner and 
Kukolich. However, we should note that the $\mathrm{C}_{1}-\mathrm{C}_{2}$ predicted bond length, which should have a partial triple-bond character, is $0.019 \AA$ off from the experimental measurement. The latter is not present in the CASSCF structure. PHF better reproduces other geometrical parameters than in the $p$ - and $m$-isomers.

\section{Vibrational frequencies}

In the following, we discuss the frequencies of $p$ benzyne, where strong correlation effects are most significant. The PHF vibrational spectra of the other benzyne isomers are presented in Appendix B. We enumerate the harmonic frequencies according to their irreducible representations in Table II.

The difficulty in the calculation of the harmonic spectrum of $p$-benzyne by single reference methods has been first outlined by Crawford et al. ${ }^{43}$ Specifically, the frequencies $b_{1 u} / 12$ and $b_{2 g} / 14$ which were found imaginary by these authors at the RHF-CCSD(T) level, are heavily dependent on a proper accounting of static correlation effects. ${ }^{47}$

The SUHF harmonic spectrum agrees with calculations using more sophisticated (and expensive) coupled-cluster $(\mathrm{CC})^{47}$ techniques, which go beyond single reference wave functions. Comparing to CASSCF, one may notice that most PHF frequencies are below the corresponding CASSCF values, which is known to overestimate the harmonic

TABLE II. Vibrational frequencies (in $\mathrm{cm}^{-1}$ ) of $p$-benzyne as predicted by SUHF, KSUHF, and other methods.

\begin{tabular}{|c|c|c|c|c|c|c|}
\hline Sym. & No. & Expt. $^{\mathrm{a}}$ & CASSCF & SUHF & KSUHF & RMR-CCSD $(T)^{b}$ \\
\hline \multirow[t]{5}{*}{$a_{g}$} & 1 & & 3352 & 3318 & 3342 & 3236 \\
\hline & 2 & & 1537 & 1456 & 1477 & 1385 \\
\hline & 3 & & 1219 & 1074 & 1186 & 1167 \\
\hline & 4 & 990 & 1040 & 1000 & 1008 & 1023 \\
\hline & 5 & 635 & 647 & 619 & 623 & 647 \\
\hline \multirow[t]{2}{*}{$a_{u}$} & 6 & & 962 & 936 & 942 & 924 \\
\hline & 7 & & 431 & 383 & 384 & 429 \\
\hline$b_{1 g}$ & 8 & & 771 & 759 & 768 & 692 \\
\hline \multirow[t]{4}{*}{$b_{1 u}$} & 9 & & 3334 & 3302 & 3326 & 3215 \\
\hline & 10 & 1403 & 1565 & 1526 & 1544 & 1468 \\
\hline & 11 & 978 & 1088 & 1062 & 1075 & 1063 \\
\hline & 12 & 918 & 1039 & 1007 & 1016 & 869 \\
\hline \multirow[t]{2}{*}{$b_{2 g}$} & 13 & & 933 & 914 & 923 & 857 \\
\hline & 14 & & 657 & 658 & 660 & 547 \\
\hline \multirow[t]{4}{*}{$b_{2 u}$} & 15 & & 3350 & 3315 & 3339 & 3230 \\
\hline & 16 & 1331 & 1407 & 1409 & 1427 & 1341 \\
\hline & 17 & 1207 & 1265 & 1327 & 1346 & 1223 \\
\hline & 18 & & 1092 & 1071 & 1080 & 1040 \\
\hline \multirow[t]{4}{*}{$b_{3 g}$} & 19 & & 3334 & 3301 & 3326 & 3217 \\
\hline & 20 & & 1694 & 1628 & 1643 & 1665 \\
\hline & 21 & & 1372 & 1351 & 1365 & 1290 \\
\hline & 22 & & 625 & 619 & 622 & 573 \\
\hline \multirow[t]{2}{*}{$b_{3 u}$} & 23 & 721 & 777 & 770 & 776 & 758 \\
\hline & 24 & 435 & 460 & 449 & 449 & 500 \\
\hline
\end{tabular}

${ }^{a}$ See Refs. 52 and 56.

${ }^{\mathrm{b}}$ From Ref. 47 (see Table I (4R RMR-CCSD)). frequencies. The case of $p$-benzyne thus clearly illustrates that the proper accounting of static correlations is a necessary condition to describe the shape of the PES in the benzyne isomers.

\section{CONCLUSIONS}

We have derived and implemented analytic energy gradients for the projected HF method, which we hope will facilitate PHF applications in quantum chemistry. The derivative expressions do not require the solution of CPHF-like equations and have several analogies with derivatives in standard HF theory. Higher order derivatives could be produced in a similar way; however, the formulation of Coupled-Perturbed equations for PHF will then be necessary.

Our test calculations show that PHF is capable of capturing effects of static correlation and predicts qualitatively correct potential energy surfaces. However, the amount of recovered correlation energy, and thus the quality of the potential energy surface, varies for different systems.

\section{ACKNOWLEDGMENTS}

This work was supported by the Department of Energy, Office of Basic Energy Sciences, Grant No. DE-FG0209ER16053 and DOE-CMCSN (DE-SC0006650. G.E.S. is a Welch Foundation Chair (C-0036).

\section{APPENDIX A: CONNECTION WITH CONVENTIONAL HF GRADIENT}

We show here that the PHF energy derivative (Eq. (28)) reduces to the standard HF derivative derived by Pulay ${ }^{57}$ in the case of a trivial projection operator $\hat{P}=\hat{I}$. In this case, the integral and summation in Eq. (28) collapse to a single point. We additionally have

$$
R(\theta) \rightarrow S, \quad n(\theta) \rightarrow 1, \quad h(\theta) \rightarrow E .
$$

The first two terms in Eq. (28) are just traces of the density matrix with the derivatives of atomic integrals found in the HF derivative expression. The third term identically vanishes. We also have

$$
\rho(\theta) \rightarrow \rho=C C^{\dagger} .
$$

The fourth term in Eq. (28) can then be recognized as a product of the HF Fock and density matrices

$$
\begin{aligned}
& \sum_{\mu \nu \lambda \sigma} S_{\mu \nu}^{x} \rho_{\nu \lambda}\left[\langle\lambda|h| \sigma\rangle+G_{\lambda \sigma}\right] \rho_{\sigma \mu} \\
& \quad=\sum_{\mu \nu \lambda \sigma} S_{\mu \nu}^{x} \rho_{\nu \lambda} F_{\lambda \sigma} \rho_{\sigma \nu} \\
& \quad=\sum_{\mu \nu \lambda i j} S_{\mu \nu}^{x} C_{\lambda i} \epsilon_{i j} C_{j \mu}^{\dagger}=\operatorname{Tr}\left(S^{x} W\right) .
\end{aligned}
$$

Here, we have used the HF equations $(F C=S C \epsilon)$, satisfied at convergence, as well as the orthonormality condition among the occupied orbitals. The matrix $W$ is the so-called energy-weighted density matrix in the last equality. Putting 
TABLE III. SUHF natural occupations and UHF charge natural occupations in the ground state of benzyne isomers on SUHF geometries.

\begin{tabular}{|c|c|c|c|c|c|c|}
\hline \multirow[b]{2}{*}{ No. } & \multicolumn{2}{|c|}{$p$-benzyne } & \multicolumn{2}{|c|}{$m$-benzyne } & \multicolumn{2}{|c|}{$o$-benzyne } \\
\hline & UHF & SUHF & UHF & SUHF & UHF & SUHF \\
\hline 16 & 1.996 & 1.990 & 1.998 & 1.992 & 1.996 & 1.992 \\
\hline 17 & 1.952 & 1.937 & 1.984 & 1.961 & 1.961 & 1.951 \\
\hline 18 & 1.813 & 1.803 & 1.928 & 1.875 & 1.850 & 1.860 \\
\hline 19 & 1.800 & 1.801 & 1.891 & 1.843 & 1.819 & 1.838 \\
\hline 20 & 1.080 & 1.161 & 1.294 & 1.553 & 1.498 & 1.726 \\
\hline 21 & 0.920 & 0.839 & 0.706 & 0.447 & 0.502 & 0.274 \\
\hline 22 & 0.200 & 0.199 & 0.109 & 0.157 & 0.181 & 0.162 \\
\hline 23 & 0.187 & 0.197 & 0.072 & 0.125 & 0.149 & 0.141 \\
\hline 24 & 0.048 & 0.063 & 0.016 & 0.039 & 0.039 & 0.049 \\
\hline 25 & 0.004 & 0.010 & 0.003 & 0.008 & 0.004 & 0.008 \\
\hline
\end{tabular}

all the pieces together, we recover the familiar HF derivative expression

$$
\frac{\partial E}{\partial x}=\sum_{\mu \nu}\left[\langle\mu|h| \nu\rangle^{x} \rho_{\nu \mu}+G_{\mu \nu}^{x}(\theta) \rho_{\nu \mu}\right]-\sum_{\mu \nu} S_{\mu \nu}^{x} W_{\nu \mu}
$$

\section{APPENDIX B: NATURAL ORBITAL OCCUPATIONS}

We list here the natural orbital occupations of our final SUHF wave functions, as well as the charge natu-

TABLE IV. Vibrational frequencies (in $\mathrm{cm}^{-1}$ ) of $m$-benzyne as obtained with SUHF, SGHF, and other methods. See discussion in the text regarding the SUHF geometry.

\begin{tabular}{lcccrcc}
\hline \hline Sym. & No. & Expt. $^{\mathrm{a}}$ & CASSCF & SUHF & SGHF & RMR-CCSD(T) $^{\mathrm{b}}$ \\
\hline$a_{1}$ & 1 & 3037 & 3399 & 3388 & 3338 & 3302 \\
& 2 & & 3348 & 3323 & 3275 & 3228 \\
& 3 & & 3313 & 3284 & 3248 & 3160 \\
& 4 & & 1722 & 1762 & 1662 & 1656 \\
& 5 & 1402 & 1516 & 1525 & 1477 & 1447 \\
& 6 & & 1155 & 1161 & 1124 & 1101 \\
& 7 & & 1069 & 1087 & 1038 & 1025 \\
& 8 & & 949 & 937 & 937 & 836 \\
& 9 & 367 & 506 & 488 & 506 & 419 \\
$a_{2}$ & 10 & & 883 & 945 & 905 & 825 \\
& 11 & & 501 & 523 & 485 & 492 \\
$b_{1}$ & 12 & & 990 & 1083 & 1012 & 917 \\
& 13 & 824 & 870 & 940 & 880 & 872 \\
& 14 & 751 & 789 & 841 & 794 & 742 \\
& 15 & 561 & 604 & 664 & 617 & 513 \\
& 16 & 362 & 410 & 432 & 408 & 377 \\
$b_{2}$ & 17 & & 3341 & 3287 & 3255 & 3196 \\
& 18 & 1486 & 1612 & 1651 & 1572 & 1372 \\
& 19 & & 1493 & 1504 & 1473 & 1519 \\
& 20 & & 1363 & 1367 & 1342 & 1255 \\
& 21 & & 1286 & 1248 & 1274 & 1382 \\
& 22 & & 1181 & 1084 & 1175 & 1180 \\
& 23 & 936 & 1016 & 650 & 1000 & 969 \\
& 24 & 547 & 628 & $119 i$ & 612 & 616 \\
\hline \hline & & & & & &
\end{tabular}

${ }^{\mathrm{a}}$ From Refs. 59 and 60.

${ }^{\mathrm{b}}$ See Table VI of Ref. 47.
TABLE V. Vibrational frequencies $\left(\right.$ in $\mathrm{cm}^{-1}$ ) of $o$-benzyne as obtained with SUHF and other methods.

\begin{tabular}{lccccc}
\hline \hline Sym. & No. & Expt. $^{\text {a }}$ & CASSCF & SUHF & CCSD(T)/cc-pVTZ \\
\hline$a_{1}$ & 1 & 3094 & 3363 & 3327 & 3220 \\
& 2 & 3071 & 3331 & 3307 & 3191 \\
& 3 & 1846 & 1924 & 1774 & 1904 \\
& 4 & 1415 & 1568 & 1549 & 1477 \\
& 5 & 1271 & 1397 & 1378 & 1318 \\
& 6 & 1055 & 1219 & 1208 & 1154 \\
& 7 & 1039 & 1066 & 1068 & 1055 \\
& 8 & 982 & 1027 & 1009 & 996 \\
& 9 & 589 & 646 & 631 & 606 \\
$a_{2}$ & 10 & & 997 & 1007 & 949 \\
& 11 & & 869 & 878 & 862 \\
& 12 & & 638 & 656 & 592 \\
& 13 & & 451 & 465 & 440 \\
$b_{1}$ & 14 & 838 & 952 & 956 & 914 \\
& 15 & 737 & 763 & 766 & 746 \\
& 16 & 388 & 418 & 394 & 387 \\
$b_{2}$ & 17 & 3086 & 3360 & 3322 & 3216 \\
& 18 & 3049 & 3314 & 3292 & 3174 \\
& 19 & 1451 & 1631 & 1562 & 1488 \\
& 20 & 1394 & 1513 & 1497 & 1418 \\
& 21 & 1307 & 1319 & 1303 & 1261 \\
& 22 & 1094 & 1182 & 1136 & 1107 \\
& 23 & 849 & 949 & 953 & 849 \\
\hline & 24 & 472 & 563 & 579 & 462 \\
\hline \multirow{6}{*}{2} & & & & &
\end{tabular}

${ }^{\mathrm{a}}$ From Ref. 61

${ }^{\mathrm{b}}$ From Ref. 58

ral orbital occupations of UHF. The orbital occupations in Table III justify our choice of the active space in CASSCF calculations.

\section{APPENDIX C: VIBRATIONAL FREQUENCIES OF $M$ - AND O-BENZYNES}

The vibrational frequencies of $m$ - and $o$-benzyne are less illustrative than in the case of $p$-benzyne, as these isomers display less strong correlation effects. Both molecules have $C_{2 v}$ symmetric equilibrium geometries.

For $m$-benzyne, we considered the minimum in the $C_{2 v}$ surface which, as can be seen from Table. IV, is not predicted to be a true minimum by SUHF (there is one imaginary frequency). In fact, the symmetry of the SUHF actual minimum is reduced to $C_{s}$. Consequently, some normal modes differ significantly from the corresponding CASSCF and experimental values (cf. modes $b_{2} / 24, b_{2} / 23$ ). However, SGHF predicts an expected $C_{2 v}$ minimum and improves the harmonic spectrum.

$o$-benzyne exibits the lowest biradical character of all three isomers, as we discussed above. Consequently, the ground state wave function of $o$-benzyne can be well described with single-reference methods. Indeed, singlereference CC calculations ${ }^{58}$ reproduce the experimental vibrational spectrum quite accurately. The SUHF frequencies are of CASSCF quality (most frequencies have actually smaller errors) (Table V). 
${ }^{1}$ B. O. Roos, P. R. Taylor, and P. E. Siegbahn, Chem. Phys. 48, 157 (1980).

${ }^{2}$ J. M. Bofill and P. Pulay, J. Chem. Phys. 90, 3637 (1989).

${ }^{3}$ P. Pulay and T. P. Hamilton, J. Chem. Phys. 88, 4926 (1988).

${ }^{4}$ P. O. Dral and T. Clark, J. Phys. Chem. A 115, 11303 (2011).

${ }^{5}$ C. A. Jiménez-Hoyos, T. M. Henderson, T. Tsuchimochi, and G. E. Scuseria, J. Chem. Phys. 136, 164109 (2012).

${ }^{6}$ R. E. Peierls and J. Yoccoz, Proc. Phys. Soc. A 70, 381 (1957).

${ }^{7}$ H. B. Schlegel, J. Chem. Phys. 84, 4530 (1986).

${ }^{8}$ K. Yamaguchi, Y. Yoshioka, T. Takatsuka, and T. Fueno, Theor. Chim. Acta 48, 185 (1978).

${ }^{9}$ Y. Cui, I. W. Bulik, C. A. Jiménez-Hoyos, T. M. Henderson, and G. E. Scuseria, J. Chem. Phys. 139, 154107 (2013).

${ }^{10}$ P.-O. Löwdin, Phys. Rev. 97, 1509 (1955)

${ }^{11}$ C. A. Jiménez-Hoyos, T. M. Henderson, and G. E. Scuseria, J. Chem. Theory Comput. 7, 2667 (2011).

${ }^{12}$ H. Fukutome, Int. J. Quantum Chem. 20, 955 (1981).

${ }^{13}$ J. L. Stuber, and J. Paldus, in Fundamental World of Quantum Chemistry, A Tribute Volume to the Memory of Per-Olov Löwdin, edited by E. J. Brändas and E. S. Kryachko (Kluwer Academic Publishers, Dordrecht, 2003), Vol. 1, pp. 67-139.

${ }^{14}$ G. E. Scuseria, C. A. Jiménez-Hoyos, T. M. Henderson, K. Samanta, and J. K. Ellis, J. Chem. Phys. 135, 124108 (2011).

${ }^{15}$ R. Rodríguez-Guzmán, C. A. Jiménez-Hoyos, R. Schutski, and G. E. Scuseria, Phys. Rev. B 87, 235129 (2013).

${ }^{16}$ R. Rodríguez-Guzmán, K. W. Schmid, C. A. Jiménez-Hoyos, and G. E. Scuseria, Phys. Rev. B 85, 245130 (2012).

${ }^{17}$ D. L. Strout and G. E. Scuseria, J. Chem. Phys. 102, 8448 (1995).

${ }^{18}$ I. Mayer and M. Kertész, Int. J. Quantum Chem. 10, 961 (1976).

${ }^{19}$ C. A. Jiménez-Hoyos, R. Rodríguez-Guzmán, and G. E. Scuseria, J. Chem. Phys. 139, 204102 (2013).

${ }^{20}$ H. Shi, C. A. Jiménez-Hoyos, R. Rodríguez-Guzmán, G. E. Scuseria, and S. Zhang, Phys. Rev. B 89, 125129 (2014).

${ }^{21}$ N. C. Handy and J. E. Rice, in Quantum Chemistry: Basic Aspects, Actual Trends, edited by R. Carbó (Elsevier, Amsterdam, 1989), pp. $145-167$.

${ }^{22}$ I. Mayer, Adv. Quantum Chem. 12, 189 (1980).

${ }^{23}$ K. Schmid and F. Grümmer, Z. Phys. A 292, 15 (1979).

${ }^{24}$ K. W. Schmid, F. Grümmer, and A. Faessler, Phys. Rev. C 29, 291 (1984).

${ }^{25}$ E. Wigner Group Theory and Its Application to the Quantum Mechanics of Atomic Spectra, Pure and Applied Physics, A Series of Monographs and Textbooks Vol. 5, edited by H. S. W. Massey (Academic Press, New York, 1959).

${ }^{26}$ P. Ring and P. Schuck, The Nuclear Many-Body Problem (Springer-Verlag, New York, 1980).

${ }^{27}$ J.-P. Blaizot and G. Ripka, Quantum Theory of Finite Systems (MIT Press, Cambridge, 1986).

${ }^{28}$ S. Bratoz, Colloq. Int. CNRS 82, 287 (1958).

${ }^{29}$ P. Pulay, Mol. Phys. 17, 197 (1969).

${ }^{30}$ J. Gerratt and I. M. Mills, J. Chem. Phys. 49, 1719 (1968).

${ }^{31}$ P. Pulay and H. F. Schaefer III, in Modern Theoretical Chemistry (Plenum, New York, 1977), Vol. 4, p. 153.
${ }^{32}$ M. J. Frisch, G. W. Trucks, H. B. Schlegel et al., GAUSSIAN Development Version, Revision H.01, Gaussian Inc., Wallingford, CT, 2012.

${ }^{33}$ M. Rosenberg and F. Martino, J. Chem. Phys. 63, 5354 (1975).

${ }^{34}$ V. Klimo and J. Tin̆o, Mol. Phys. 35, 1777 (1978).

${ }^{35}$ P. B. Karadakov and D. L. Cooper, Int. J. Quantum Chem. 109, 2447 (2009).

${ }^{36}$ K. Samanta, C. A. Jiménez-Hoyos, and G. E. Scuseria, J. Chem. Theory Comput. 8, 4944 (2012).

${ }^{37}$ P. Rivero, C. A. Jiménez-Hoyos, and G. E. Scuseria, J. Phys. Chem. A 117, 8073 (2013).

${ }^{38}$ P. Rivero, C. A. Jiménez-Hoyos, and G. E. Scuseria, J. Phys. Chem. B 117, 12750 (2013).

${ }^{39}$ W. T. Borden, Diradicals (Wiley, New York, 1982).

${ }^{40}$ L. V. Slipchenko and A. I. Krylov, J. Chem. Phys. 117, 4694 (2002).

${ }^{41}$ W. Sander, Accounts Chem. Res. 32, 669 (1999).

${ }^{42}$ H. H. Wenk, M. Winkler, and W. Sander, Angew. Chem., Int. Ed. 42, 502 (2003).

${ }^{43}$ T. D. Crawford, E. Kraka, J. F. Stanton, and D. Cremer, J. Chem. Phys. 114, 10638 (2001)

${ }^{44} \mathrm{X}$. Li and J. Paldus, J. Chem. Phys. 129, 174101 (2008).

${ }^{45}$ F. A. Evangelista, W. D. Allen, and H. F. Schaefer III, J. Chem. Phys. 127, 024102 (2007).

${ }^{46}$ C. E. Smith, T. D. Crawford, and D. Cremer, J. Chem. Phys. 122, 174309 (2005).

${ }^{47}$ X. Li and J. Paldus, J. Chem. Phys. 132, 114103 (2010).

${ }^{48}$ Y. Shao, M. Head-Gordon, and A. I. Krylov, J. Chem. Phys. 118, 4807 (2003).

${ }^{49}$ S. G. Wierschke, J. J. Nash, and R. R. Squires, J. Am. Chem. Soc. 115, 11958 (1993).

${ }^{50}$ C. J. Cramer, J. J. Nash, and R. R. Squires, Chem. Phys. Lett. 277, 311 (1997).

${ }^{51}$ D. G. Leopold, A. E. S. Miller, and W. C. Lineberger, J. Am. Chem. Soc. 108, 1379 (1986).

${ }^{52}$ P. G. Wenthold, R. R. Squires, and W. C. Lineberger, J. Am. Chem. Soc. 120, 5279 (1998).

${ }^{53}$ F. A. Hamprecht, A. J. Cohen, D. J. Tozer, and N. C. Handy, J. Chem. Phys. 109, 6264 (1998).

${ }^{54}$ J. E. Peralta, G. E. Scuseria, and M. J. Frisch, Phys. Rev. B 75, 125119 (2007).

${ }^{55}$ E. Kraka and D. Cremer, Chem. Phys. Lett. 216, 333 (1993).

${ }^{56}$ R. Marquardt, A. Balster, W. Sander, E. Kraka, D. Cremer, and J. G. Radziszewski, Angew. Chem., Int. Ed. 37, 955 (1998).

${ }^{57}$ P. Pulay, in Applications of Electronic Structure Theory, edited by H. F. Schaefer III (Plenum Press, New York, 1977), pp. 153-185.

${ }^{58}$ X. Zhang, A. T. Maccarone, M. R. Nimlos, S. Kato, V. M. Bierbaum, G. B. Ellison, B. Ruscic, A. C. Simmonett, W. D. Allen, and H. F. Schaefer III, J. Chem. Phys. 126, 044312 (2007).

${ }^{59}$ R. Marquardt, W. Sander, and E. Kraka, Angew. Chem., Int. Ed. 35, 746 (1996).

${ }^{60}$ W. Sander, M. Exner, M. Winkler, A. Balster, A. Hjerpe, E. Kraka, and D. Cremer, J. Am. Chem. Soc. 124, 13072 (2002).

${ }^{61}$ J. G. Radziszewski, B. A. Hess, Jr., and R. Zahradnik, J. Am. Chem. Soc. 114, 52 (1992). 